\title{
\begin{tabular}{l|l} 
Mibraries & DSpace@MIT
\end{tabular}
}

\author{
MIT Open Access Articles
}

\author{
Search for Muon Neutrino and \\ Antineutrino Disappearance in MiniBooNE
}

The MIT Faculty has made this article openly available. Please share how this access benefits you. Your story matters.

Citation: MiniBooNE Collaboration et al. "Search for Muon Neutrino and Antineutrino Disappearance in MiniBooNE." Physical Review Letters 103.6 (2009): 061802. () 2009 The American Physical Society.

As Published: http://dx.doi.org/10.1103/PhysRevLett.103.061802

Publisher: American Physical Society

Persistent URL: http://hdl.handle.net/1721.1/53415

Version: Final published version: final published article, as it appeared in a journal, conference proceedings, or other formally published context

Terms of Use: Article is made available in accordance with the publisher's policy and may be subject to US copyright law. Please refer to the publisher's site for terms of use. 


\section{Search for Muon Neutrino and Antineutrino Disappearance in MiniBooNE}

A. A. Aguilar-Arevalo, ${ }^{14}$ C.E. Anderson, ${ }^{19}$ A. O. Bazarko, ${ }^{16}$ S. J. Brice, ${ }^{7}$ B. C. Brown, ${ }^{7}$ L. Bugel,${ }^{5}$ J. Cao, ${ }^{15}$ L. Coney, ${ }^{5}$ J. M. Conrad, ${ }^{5,13}$ D. C. Cox,${ }^{10}$ A. Curioni, ${ }^{19}$ Z. Djurcic,${ }^{5}$ D. A. Finley,${ }^{7}$ B. T. Fleming, ${ }^{19}$ R. Ford, ${ }^{7}$ F. G. Garcia, ${ }^{7}$ G. T. Garvey, ${ }^{11}$ J. Grange, ${ }^{8}$ C. Green,,${ }^{7,11}$ J. A. Green, ${ }^{10,11}$ T. L. Hart, ${ }^{4}$ E. Hawker,,${ }^{311}$ R. Imlay, ${ }^{12}$ R. A. Johnson, ${ }^{3}$ G. Karagiorgi, ${ }^{5,13}$ P. Kasper, ${ }^{7}$ T. Katori, ${ }^{10,13}$ T. Kobilarcik, ${ }^{7}$ I. Kourbanis, ${ }^{7}$ S. Koutsoliotas, ${ }^{2}$ E. M. Laird, ${ }^{16}$ S. K. Linden, ${ }^{19}$ J. M. Link,${ }^{18}$ Y. Liu, ${ }^{15}$ Y. Liu, ${ }^{1}$ W. C. Louis, ${ }^{11}$ K. B. M. Mahn, ${ }^{5}$ W. Marsh, ${ }^{7}$ C. Mauger, ${ }^{11}$ V. T. McGary, ${ }^{5,13}$ G. McGregor, ${ }^{11}$ W. Metcalf, ${ }^{12}$ P. D. Meyers ${ }^{16}$ F. Mills, ${ }^{7}$ G. B. Mills, ${ }^{11}$ J. Monroe, ${ }^{5}$ C. D. Moore,${ }^{7}$ J. Mousseau, ${ }^{8}$ R. H. Nelson,${ }^{4}$ P. Nienaber,${ }^{17}$ J. A. Nowak, ${ }^{12}$ B. Osmanov ${ }^{8}$ S. Ouedraogo, ${ }^{12}$ R. B. Patterson, ${ }^{16}$ Z. Pavlovic, ${ }^{11}$ D. Perevalov, ${ }^{1}$ C. C. Polly, ${ }^{9,10}$ E. Prebys, ${ }^{7}$ J. L. Raaf,${ }^{3}$ H. Ray, ${ }^{8,11}$ B. P. Roe, ${ }^{15}$ A. D. Russell, ${ }^{7}$ V. Sandberg, ${ }^{11}$ R. Schirato, ${ }^{11}$ D. Schmitz, ${ }^{5}$ M. H. Shaevitz, ${ }^{5}$ F. C. Shoemaker,${ }^{16, *}$ D. Smith, ${ }^{6}$ M. Soderberg, ${ }^{19}$ M. Sorel,${ }^{5, \dagger}$ P. Spentzouris, ${ }^{7}$ J. Spitz, ${ }^{19}$ I. Stancu, ${ }^{1}$ R. J. Stefanski, ${ }^{7}$ M. Sung,${ }^{12}$ H. A. Tanaka, ${ }^{16}$ R. Tayloe, ${ }^{10}$ M. Tzanov, ${ }^{4}$ R. G. Van de Water, ${ }^{11}$ M. O. Wascko, ${ }^{12,}$ D. H. White, ${ }^{11}$ M. J. Wilking, ${ }^{4}$ H. J. Yang, ${ }^{15}$ G. P. Zeller, ${ }^{5,11}$ and E. D. Zimmerman ${ }^{4}$

(MiniBooNE Collaboration)

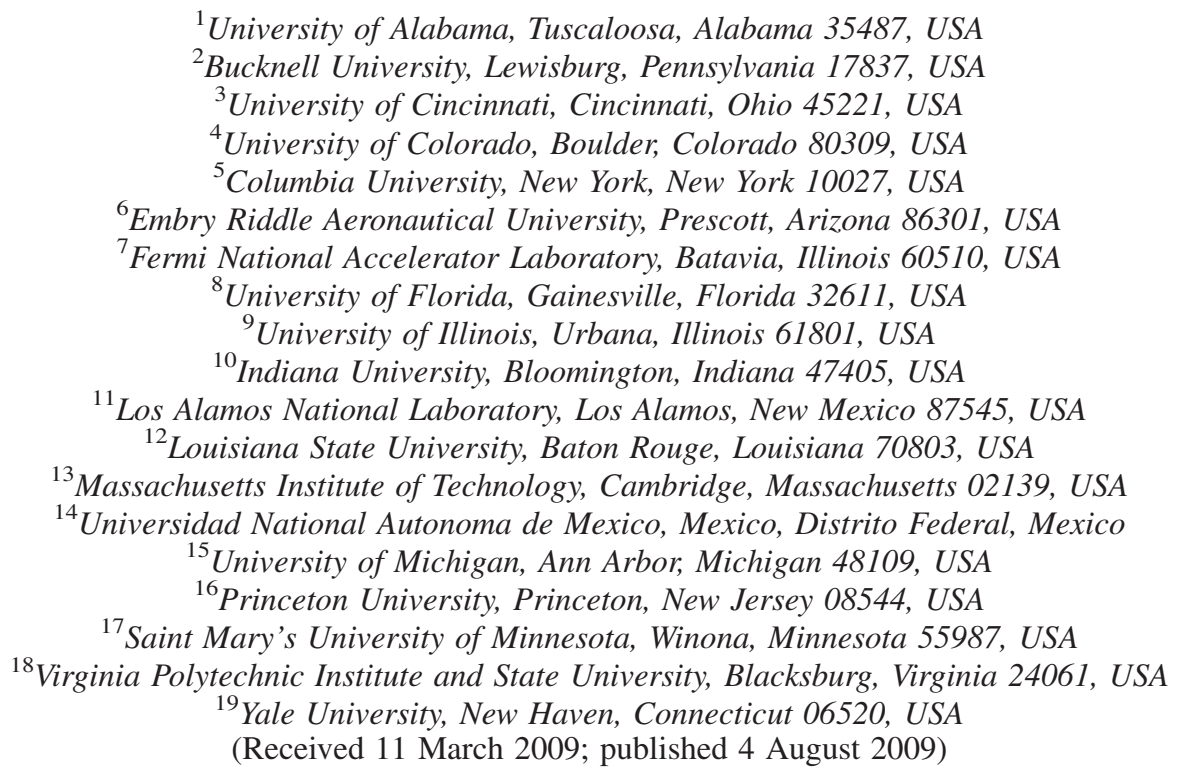

The MiniBooNE Collaboration reports a search for $\nu_{\mu}$ and $\bar{\nu}_{\mu}$ disappearance in the $\Delta m^{2}$ region of 0.5-40 eV $\mathrm{eV}^{2}$. These measurements are important for constraining models with extra types of neutrinos, extra dimensions, and CPT violation. Fits to the shape of the $\nu_{\mu}$ and $\bar{\nu}_{\mu}$ energy spectra reveal no evidence for disappearance at the $90 \%$ confidence level (C.L.) in either mode. The test of $\bar{\nu}_{\mu}$ disappearance probes a region below $\Delta m^{2}=40 \mathrm{eV}^{2}$ never explored before.

DOI: 10.1103/PhysRevLett.103.061802

Neutrino oscillations have been observed and confirmed at mass splittings $\left(\Delta m^{2}\right)$ of $\sim 10^{-5} \mathrm{eV}^{2}$ and $\sim 10^{-3} \mathrm{eV}^{2}$, called the "solar" and "atmospheric" oscillations, respectively. The observed mixing is consistent with three generations of neutrinos and a unitary mixing matrix. Complicating this picture, the Liquid Scintillator Neutrino Detector (LSND) experiment observed an excess of $\bar{\nu}_{e}$ in a $\bar{\nu}_{\mu}$ beam [1], indicating a possible third $\Delta m^{2}$ around $1 \mathrm{eV}^{2}$ thus requiring more than three neutrino generations or other exotic physics. Recently, the MiniBooNE experiment [2] excluded two-neutrino
PACS numbers: 14.60.St, 14.60.Lm, 14.60.Pq appearance-only oscillations (98\% C.L.) as an explanation of the LSND excess if oscillations of neutrinos and antineutrinos are the same.

Exotic physics models [3-6], including sterile neutrinos, extra dimensions, and $C P T$ violation, have been proposed to explain the LSND observation. Some of these models can also accommodate the MiniBooNE $\nu_{e}$ appearance oscillation results. These models are testable with measurements of $\nu_{\mu}$ and $\bar{\nu}_{\mu}$ disappearance which constrain any nonstandard oscillations of $\stackrel{(-)}{\nu}_{\mu} \rightarrow \stackrel{(-)}{\nu}_{x}$. As described in this Letter, the MiniBooNE Collaboration has performed 
searches for $\nu_{\mu}$ and $\bar{\nu}_{\mu}$ disappearance which probe a region of interest, $\Delta m^{2}=0.5-40 \mathrm{eV}^{2}$, not covered by two previous disappearance experiments, CCFR $\left(\nu_{\mu}\right.$ and $\bar{\nu}_{\mu}$ ) [7] and CDHS ( $\nu_{\mu}$ only) [8]. Unless otherwise stated, all statements about neutrinos hold true also for antineutrinos.

For the MiniBooNE experimental setup, the detector [9] is located at a fixed distance from the neutrino source. In this case, $\nu_{\mu}$ disappearance due to oscillations has a distinct signature as a function of neutrino energy, because neutrinos with different energies oscillate with different probabilities for the same distance traveled. Disappearance would be observable either via a deficit of events (normalization) or, alternatively, via a distortion of the neutrino energy spectrum (shape), or both (normalization + shape). The absolute normalization uncertainties in a single detector experiment such as MiniBooNE are large, so a shapeonly disappearance fit is performed. The $\stackrel{(-)}{\nu}_{\mu}$ flux to the MiniBooNE detector is provided by the Fermilab Booster Neutrino Beam, produced by $8 \mathrm{GeV}$ protons incident on a $1 \mathrm{~cm}$ diameter, $71 \mathrm{~cm}$ long (1.7 interaction length) beryllium target surrounded by a magnetic horn pulsed at $174 \mathrm{kA}$. The horn uses a positive current to focus $\pi^{+}$ and $K^{+}$mesons for the neutrino mode sample, and a negative current to focus $\pi^{-}$and $K^{-}$for the antineutrino mode sample. The mesons that pass through a $60 \mathrm{~cm}$ diameter collimator $259 \mathrm{~cm}$ downstream of the target decay in a $50 \mathrm{~m}$ long tunnel to produce the $\stackrel{(-)}{\nu}_{\mu}$ beam. The Booster Neutrino Beam flux [10] is determined using a GEANT4 [11] based beam simulation which has been further modified to include updated $p$-Be particle production data $[12,13]$.

The distance from the proton interaction target to the MiniBooNE detector [9] is $541 \mathrm{~m}$. The MiniBooNE detector is a $12 \mathrm{~m}$ diameter spherical tank filled with 800 tons of mineral oil $\left(\mathrm{CH}_{2}\right)$. The detector is separated into an inner region filled with 1280 inward-facing 8 in. photomultiplier tubes (PMTs), and an optically isolated outer region used to reject cosmic-ray induced events. Charged particles produced in neutrino interactions emit primarily Cherenkov light, though a small amount of scintillation light is also produced. Light and particle production and propagation in the MiniBooNE detector is modeled using a GEANT3 [14] based simulation, which was tuned using MiniBooNE and external data.

Neutrino interactions are simulated with the v3 NUANCE event generator [15]. Prior to selection, approximately $42 \%$ of all events in MiniBooNE are charged current quasielastic (CCQE) scattering, and $22 \%$ are charged current single charged pion production $\left(\mathrm{CC} 1 \pi^{+/-}\right)$in both the neutrino and antineutrino mode.

The search for oscillations is conducted with a sample of CCQE events because of the high statistics and purity. The reconstructed neutrino energy $\left(E_{\nu}^{\mathrm{QE}}\right)$ is calculated assuming the target nucleon is at rest:

$$
E_{\nu}^{\mathrm{QE}}=\frac{2\left(M_{n}-E_{B}\right) E_{\mu}-\left(E_{B}^{2}-2 M_{n} E_{B}+\Delta M+M_{\mu}^{2}\right)}{2\left[\left(M_{n}-E_{B}\right)-E_{\mu}+p_{\mu} \cos \theta_{\mu}\right]},
$$

where $\Delta M=M_{n}^{2}-M_{p}^{2} ; M$ indicates the muon, proton, or neutron mass with appropriate subscripts; $E_{B}$ is the nucleon binding energy; $E_{\mu}\left(p_{\mu}\right)$ is the reconstructed muon energy (momentum); and $\theta_{\mu}$ is the reconstructed muon scattering angle with respect to the neutrino beam direction. A small correction is applied in both data and simulation to account for the biasing effects of Fermi smearing. At $300 \mathrm{MeV}$, the muon energy resolution is $7 \%$ and the angular resolution is 5 degrees. The average $E_{\nu}^{\mathrm{QE}}$ resolution is $11 \%$ for CCQE events [16].

A CCQE event sample is selected by identifying a single muon in the detector and its associated decay electron, using the same criteria as in the previous measurement of CCQE model parameters on carbon [16]. Timing information from the PMTs allows the light produced by the initial neutrino interaction (first "subevent") to be separated from the light produced by the decay electron (second subevent). The timing and charge response of the PMTs is then used to reconstruct the position, kinetic energy, and direction vector of the primary particle within each subevent. Exactly two subevents are required in the analysis (the muon and its decay electron). Requiring both subevents to have fewer than six PMT hits anywhere in the veto region rejects $99.99 \%$ of all cosmic-ray interactions [9]. The first subevent must be in coincidence with a beam pulse, and have greater than 200 inner tank PMT hits, to eliminate electrons from stopped cosmic-ray muon decays. The mean emission point of the Cherenkov light along the track for the first subevent must be less than $500 \mathrm{~cm}$ from the center of the tank. The second subevent must have fewer than 200 inner PMT hits to be consistent with the decay electron energy endpoint. Finally, the distance between the electron vertex and the muon track endpoint must be less than $100 \mathrm{~cm}$, ensuring that the electron is associated with the muon track. This selection also applies to the antineutrino mode sample, as the final state nucleon is not reconstructed and the detector does not distinguish muon charge.

The selection criteria yield 190454 data events with $0<$ $E_{\nu}^{\mathrm{QE}}<1.9 \mathrm{GeV}$ for $5.58 \times 10^{20}$ protons on target in the neutrino mode sample, and 27053 data events for $3.39 \times$ $10^{20}$ protons on target in the antineutrino mode sample. According to the simulation, the neutrino mode sample is $74 \%$ pure CCQE, and the antineutrino mode sample is $70 \%$ pure CCQE. The primary background $(\sim 75 \%)$ for both the $\nu_{\mu}$ and $\bar{\nu}_{\mu}$ samples is $\mathrm{CC} 1 \pi$ events where the outgoing pion is unobserved (due, e.g., to absorption in the nucleus). Though the neutrino mode sample has $<1 \% \bar{\nu}_{\mu}$ content, the 
beam in the antineutrino mode contains a substantial contribution of $\nu_{\mu}$ due to the higher $\pi^{+}$production at the target and the higher $\nu_{\mu}$ cross section. The antineutrino mode is predicted to have $25 \% \nu_{\mu}$ content.

The CCQE cross section depends on the axial vector form factor, which is commonly assumed to have a dipole form as a function of four-momentum transfer $\left(Q^{2}\right)$ with one adjustable parameter, $M_{A}$, the axial mass. Global fits to the world's neutrino scattering data on deuterium yield $M_{A}=1.015 \mathrm{GeV}$ [17]. However, recent results from $\mathrm{K} 2 \mathrm{~K}\left(M_{A}=1.14 \pm 0.11 \mathrm{GeV}\right.$, on carbon [18]; $M_{A}=$ $1.20 \pm 0.12 \mathrm{GeV}$, on oxygen [19]) and MiniBooNE $\left(M_{A}=1.23 \pm 0.12 \mathrm{GeV}\right.$, on carbon [16]) suggest a higher effective value of $M_{A}$ for nuclear targets. In addition, the level of Pauli blocking was adjusted in the MiniBooNE analysis, using a parameter $\kappa=1.019$, to better reproduce the experimental data at low $Q^{2}$ [16]. The effect of $M_{A}$ and $\kappa$ on the $Q^{2}$ shape is pronounced, but oscillations would provide relatively little $Q^{2}$ distortion; this means that a spurious value of $M_{A}$ or $\kappa$ cannot be caused by underlying oscillations. The MiniBooNE CCQE $\nu_{\mu}$ analysis which produced a value of $M_{A}=1.23$ assumed no oscillations, and therefore those values of $M_{A}$ and $\kappa$ should not be used in a disappearance analysis of the same data set. Consequently, the lower values of $M_{A}$ and $\kappa$ are used with conservative uncertainties which span the difference between the deuterium and nuclear target results $\left(M_{A}=\right.$ $1.015 \pm 0.20 \mathrm{GeV}, \kappa=1.000 \pm 0.019)$. The disappearance limits obtained were insensitive to the values of $M_{A}$ and $\kappa$ used. With $M_{A}=1.015 \mathrm{GeV}$ and $\kappa=1.000$, the ratio of detected events to predicted events in MiniBooNE is $1.31 \pm 0.26$ for neutrinos and $1.18 \pm 0.18$ for antineutrinos. The ratio for neutrinos reported in Ref. [16] is lower because higher values of $M_{A}$ and $\kappa$ were used there.

For the disappearance search, systematic uncertainties are included for the underlying neutrino flux prediction, neutrino interaction cross section, and detector response. The method used to estimate the uncertainties due to the underlying neutrino flux prediction and detector model is identical to the method used in previous MiniBooNE results $[2,20]$. The uncertainties on the cross section include uncertainties on the $\mathrm{CCQE}$ cross section and $\mathrm{CC} 1 \pi$ background. The latter is estimated using the MiniBooNE $\mathrm{CC} 1 \pi^{+}$data sample. Systematic uncertainties produce correlated errors between $E_{\nu}^{\mathrm{QE}}$ bins that are included by developing a covariance matrix in the same manner as in previous MiniBooNE oscillation analyses [2,20]. This covariance matrix includes separate normalization and shape-only error contributions. For the shape-only disappearance search, the prediction is normalized to data, and just the shape-only covariance matrix is used.

The disappearance search uses the Pearson's $\chi^{2}$ test to determine allowed regions in the $\Delta m^{2}-\sin ^{2} 2 \theta$ plane. The $\chi^{2}$ is calculated from a comparison of the data, $d_{i}$, in the $E_{\nu}^{\mathrm{QE}}$ bin $i$, to a prediction $p_{i}\left(\Delta m^{2}, \sin ^{2} 2 \theta\right)$ for 16 bins. The prediction assumes a two-flavor $\nu_{\mu} \rightarrow \nu_{x}$ disappearance characterized by one large mass splitting $\left(\Delta m^{2} \equiv \Delta m_{h k}^{2}\right)$ between the light neutrino mass states $k$, which participate in standard three-neutrino oscillations, and $h$, the heavier neutrino state, and one oscillation amplitude $\sin ^{2} 2 \theta=$ $4\left|U_{\mu, h}\right|^{2}\left(1-\left|U_{\mu, h}\right|^{2}\right)$, where $\left|U_{\mu, h}\right|^{2}$ is the muon flavor content of the heavy state $h$ :

$$
\chi^{2}=\sum_{i, j}^{16 \text { bins }}\left(d_{i}-N p_{i}\right) M_{i j}^{-1}\left(d_{j}-N p_{j}\right)
$$

where $M_{i j}$ is the shape-only error matrix, and $N$ is a factor which normalizes the prediction to the total number of observed events in data. All neutrino events in the prediction, including the $\mathrm{CC} 1 \pi^{+}$background events, are allowed to oscillate in the fit based on the incident neutrino energy and distance traveled. The $90 \%$ C.L. limit corresponds to $\chi^{2}>23.5$ for 16 degrees of freedom (DF). The sensitivity is a fit to an unoscillated prediction including all statistical and systematic uncertainties.

The top plot of Fig. 1 (Fig. 2) shows the $E_{\nu}^{\mathrm{QE}}$ spectrum after selection cuts for the neutrino (antineutrino) data and

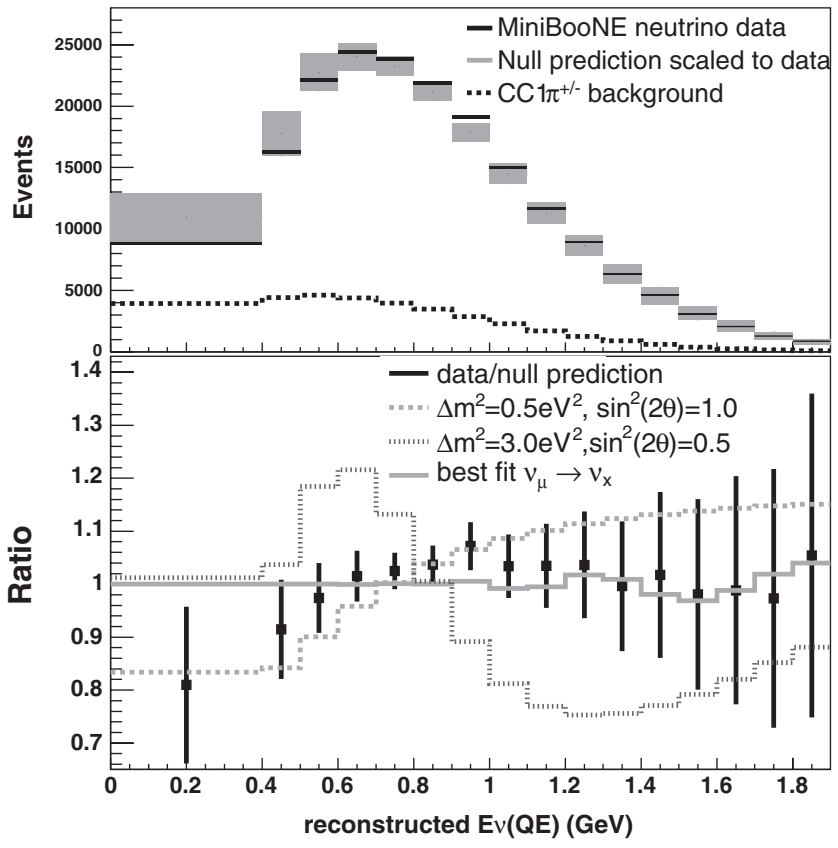

FIG. 1. The top plot shows the $E_{\nu}^{\mathrm{QE}}$ distribution for neutrino data (black) with statistical error rectangles (thickness of line indicates size of statistical error), and the prediction assuming no oscillations [area normalized to number of data events (grey)]. Attached to the prediction are the diagonal elements of the shape error matrix. The predicted $\mathrm{CC} 1 \pi$ background (dashed line) events are also shown. The background antineutrino events are negligible. The bottom plot shows the ratio of data to no oscillations (black solid line), and the ratio of no oscillations to $\Delta m^{2}=0.5 \mathrm{eV}^{2}, \sin ^{2} 2 \theta=1.0$ disappearance (dashed line), $\Delta m^{2}=3.0 \mathrm{eV}^{2}, \sin ^{2} 2 \theta=0.5$ disappearance (dotted line), and for the minimum $\chi^{2}=12.72(13 \mathrm{DF})$ at $\Delta m^{2}=17.5 \mathrm{eV}^{2}$, $\sin ^{2} 2 \theta=0.16$ (solid gray line). 
the prediction, assuming no oscillations (null hypothesis) with diagonal elements of the error matrix. The dominant systematics arise from the neutrino flux (production of $\pi^{+/-}$from $p$-Be interactions) and CCQE cross section uncertainties; uncertainties at low energy are larger because of the substantial $\mathrm{CC} 1 \pi^{+}$background and uncertainties in the CCQE cross section in this region. As shown in Fig. 1, the individual bin errors are large, but adjacent bins are nearly fully correlated. The $\chi^{2}$ between the data and the null hypothesis is 17.78 (16 DF, 34\% probability) for the neutrino mode sample, which is consistent with no oscillations at the $90 \%$ C.L. The top plot of Fig. 3 shows the $90 \%$ C.L. sensitivity and limit curves for the neutrino mode sample. The minimum $\chi^{2}=12.72(13 \mathrm{DF}, 47 \%$ probability) at $\Delta m^{2}=17.5 \mathrm{eV}^{2}, \sin ^{2} 2 \theta=0.16$. The probability distribution and number of degrees of freedom for the $\chi^{2}$ statistic are determined from an analysis of a set of simulated data samples, as suggested in Ref. [21].

The bottom plot in Fig. 1 shows the ratio of data to the null hypothesis and three oscillation scenarios. The shape distortion for $\Delta m^{2}=0.5 \mathrm{eV}^{2}$ is very different from $\Delta m^{2}=3.0 \mathrm{eV}^{2}$. The $\chi^{2}$ therefore changes rapidly as a function of $\Delta m^{2}$, resulting in rapid changes in the $90 \%$ C.L. sensitivity and limit curves (Fig. 3) for small differences in $\Delta m^{2}$. Similar features are also seen in previous disappearance analyses $[7,8]$.

The $\bar{\nu}_{\mu}$ disappearance analysis proceeds in the same manner as the $\nu_{\mu}$ analysis, except that only the $\bar{\nu}_{\mu}$ events are allowed to oscillate in the fit and the $\nu_{\mu}$ events are kept

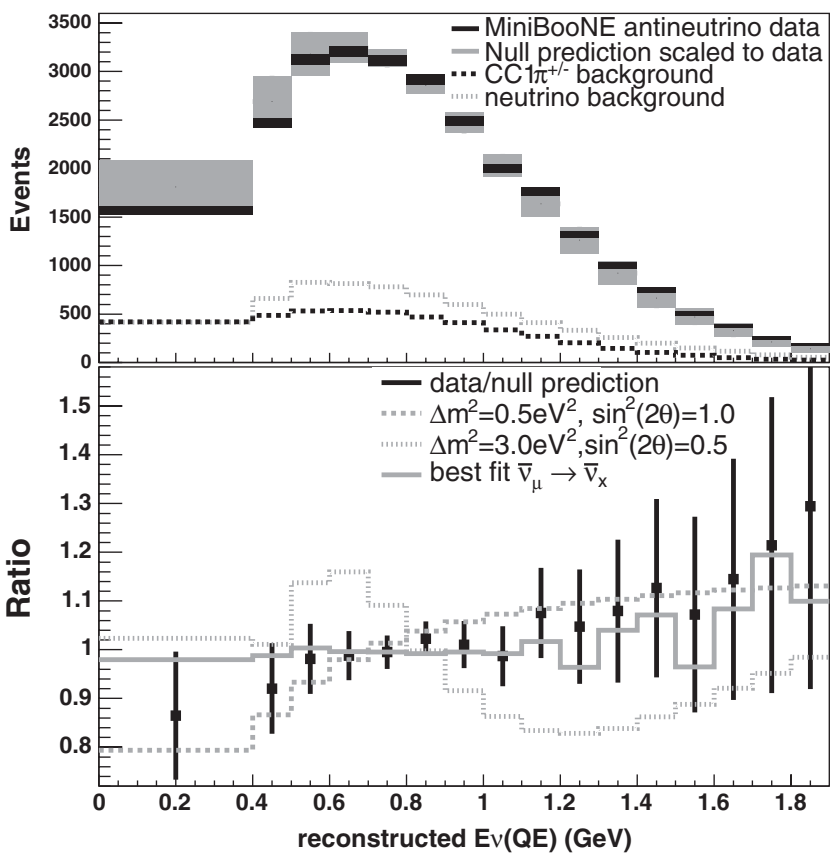

FIG. 2. Same convention as Fig. 1 for the antineutrino mode sample. The background neutrino events are also shown (dotted line in top plot). Minimum $\chi^{2}=5.43(11 \mathrm{DF})$ is at $\Delta m^{2}=$ $31.3 \mathrm{eV}^{2}, \sin ^{2} 2 \theta=0.96$. fixed. This determines the limit on a model where the $\bar{\nu}_{\mu}$ can oscillate but the $\nu_{\mu}$ cannot. A model where both $\nu_{\mu}$ and $\bar{\nu}_{\mu}$ oscillate with equal oscillation probability versus energy would produce a limit very similar to the neutrino mode limit.

During antineutrino data taking, two absorber plates inadvertently fell vertically into the decay volume at $25 \mathrm{~m}$ and were later removed, creating three distinct data taking periods with zero, one, or two absorbers in the beam line. The event rate was predicted to be $13 \%$ (20\%) lower for one (two) plate(s) in the beam. One (two) absorber plate (s) were in the beam for $16.8 \%$ (18.1\%) of the antineutrino data taking. Beam line monitoring systems indicated when each plate dropped. Because the changes to the beam line are understood, a separate simulation was run with the appropriate number of absorber plates in the beam line. Figure 2 shows the $E_{\nu}^{\mathrm{QE}}$ distribution for the antineutrino mode sample. The $\chi^{2}$ of the null hypothesis is $13.7,8.2$, $15.2,10.29$ (16 DF) for the zero, one, and two absorber plate and total data, respectively. The antineutrino mode data are also consistent with no oscillations at the $90 \%$ C.L., so the bottom plot of Fig. 3 shows the 90\% C.L. sensitivity and limit curves for the antineutrino disappear-

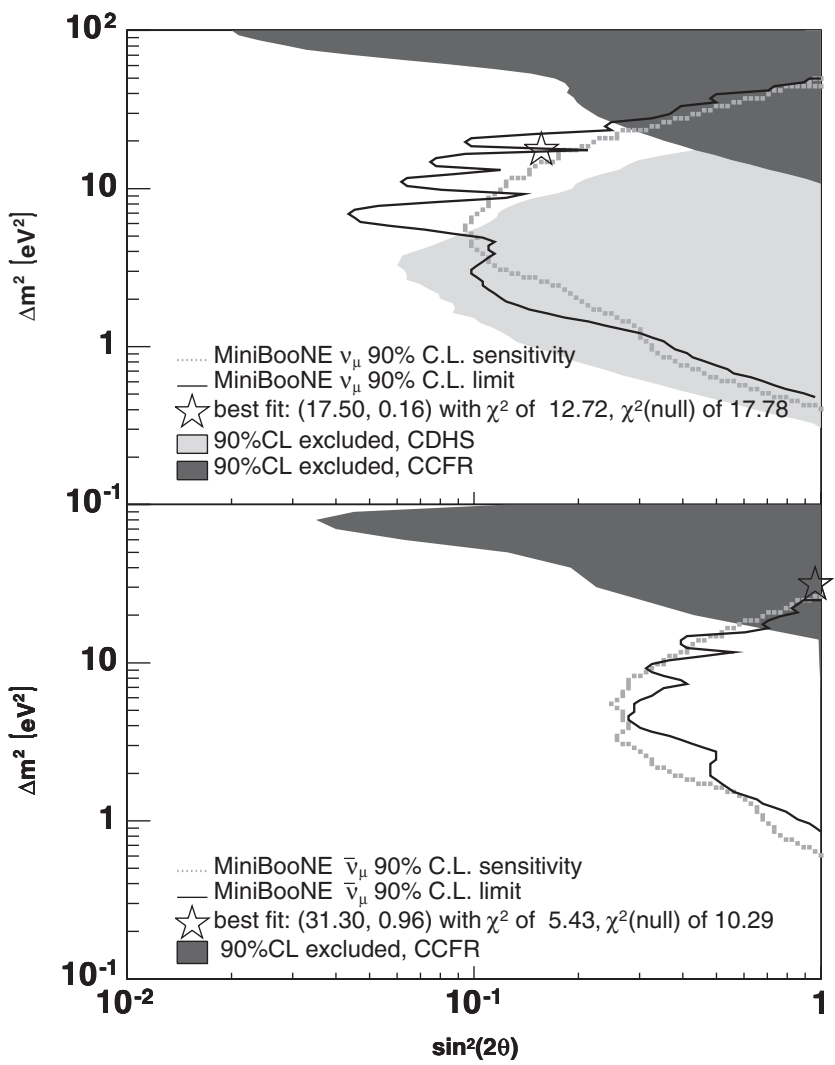

FIG. 3. The top plot shows the sensitivity (dashed line) and limit (solid line) for $90 \%$ C.L. for neutrino disappearance in MiniBooNE. Previous limits by CCFR (dark grey) and CDHS (light grey) are also shown. The bottom plot uses the same convention for antineutrino disappearance. 
ance fit to all antineutrino data; the limit curves for the individual absorber data periods were found to be consistent within errors to the total.

In addition to the two-neutrino oscillation fits described above, two $3+2$ sterile neutrino models are tested. A $3+$ 2 model assumes two heavy neutrinos mix with the lighter neutrinos, with two mass splittings and two oscillation amplitudes. Global fits to existing appearance and disappearance data yield regions of allowed $3+2$ model parameters. The best fit points in these allowed regions are tested with MiniBooNE data by forming a $\chi^{2}$ with a prediction assuming the full $3+2$ oscillation formalism with the best fit $3+2$ model parameters. The best fit $3+2$ model in Ref. [4] is consistent with both MiniBooNE $\nu_{\mu}$ and $\bar{\nu}_{\mu}$ data. However, the $\nu_{\mu}$ data are incompatible with the best fit $3+2$ model in Ref. [5] at the 90\% C.L. with $\chi^{2}=24.7(16 \mathrm{DF})$.

In summary, MiniBooNE observes no evidence for $\nu_{\mu}$ or $\bar{\nu}_{\mu}$ disappearance at the $90 \%$ C.L. in the $\Delta m^{2}$ region of $0.5-40 \mathrm{eV}^{2}$. The test of $\bar{\nu}_{\mu}$ disappearance probes a region unexplored by previous experiments.

We acknowledge the support of Fermilab, the Department of Energy, and the National Science Foundation. We thank Los Alamos National Laboratory for LDRD funding. We also acknowledge the use of Condor software in the analysis of the data.

*Deceased

${ }^{\dagger}$ Present address: IFIC, Universidad de Valencia and CSIC, Valencia 46071, Spain.

\$Present address: Imperial College, London SW7 2AZ, United Kingdom.

[1] C. Athanassopoulos et al., Phys. Rev. Lett. 75, 2650 (1995); 77, 3082 (1996); 81, 1774 (1998); A. Aguilar et al., Phys. Rev. D 64, 112007 (2001).
[2] A. Aguilar-Arevalo et al., Phys. Rev. Lett. 98, 231801 (2007).

[3] H. Pas, S. Pakvasa, and T. J. Weiler, Phys. Rev. D 72, 095017 (2005).

[4] G. Karagiorgi, A. Aguilar-Arevalo, J. M. Conrad, M. H. Shaevitz, K. Whisnant, M. Sorel, and V. Barger, Phys. Rev. D 75, 013011 (2007).

[5] M. Maltoni and T. Schwetz, Phys. Rev. D 76, 093005 (2007).

[6] A.E. Nelson and J. Walsh, Phys. Rev. D 77, 033001 (2008).

[7] I. E. Stockdale et al., Phys. Rev. Lett. 52, 1384 (1984).

[8] F. Dydak et al., Phys. Lett. B 134, 281 (1984).

[9] A. Aguilar-Arevalo et al., Nucl. Instrum. Methods Phys. Res., Sect. A 599, 28 (2009).

[10] A. Aguilar-Arevalo et al., Phys. Rev. D 79, 072002 (2009).

[11] S. Agostinelli et al., Nucl. Instrum. Methods Phys. Res., Sect. A 506, 250 (2003).

[12] M. G. Catanesi et al. (HARP Collaboration), Eur. Phys. J. C 52, 29 (2007).

[13] I. Chemakin et al., Phys. Rev. C 77, 015209 (2008); T. Abbott et al., Phys. Rev. D 45, 3906 (1992); J. V. Allaby et al., Report No. CERN 70-12, 1970; D. Dekkers et al., Phys. Rev. 137, B962 (1965); G. J. Marmer et al., Phys. Rev. 179, 1294 (1969); T. Eichten et al., Nucl. Phys. B44, 333 (1972); A. Aleshin et al., Report No. ITEP-77-80, 1977; I. A. Vorontsov et al., Report No. ITEP-88-11, 1988.

[14] CERN Program Library Long Writeup Report No. W5013, 1993.

[15] D. Casper, Nucl. Phys. B, Proc. Suppl. 112, 161 (2002).

[16] A. A. Aguilar-Arevalo et al., Phys. Rev. Lett. 100, 032301 (2008).

[17] A. Bodek, S. Avvakumov, R. Bradford, and H. Budd, J. Phys. Conf. Ser. 110, 082004 (2008).

[18] X. Espinal and F. Sanchez, AIP Conf. Proc. 967, 117 (2007).

[19] R. Gran et al., Phys. Rev. D 74, 052002 (2006).

[20] A. Aguilar-Arevalo et al., Phys. Rev. Lett. 102, 101802 (2009).

[21] G. J. Feldman and R. D. Cousins, Phys. Rev. D 57, 3873 (1998). 